\title{
A LOWER BOUND FOR RATIO OF POWER MEANS
}

\section{FENG QI, BAI-NI GUO, and LOKENATH DEBNATH}

\author{
Received 29 August 2002
}

Let $n$ and $m$ be natural numbers. Suppose that $\left\{a_{i}\right\}_{i=1}^{n+m}$ is an increasing, logarithmically convex, and positive sequence. Denote the power mean $P_{n}(r)$ for any given positive real number $r$ by $P_{n}(r)=\left((1 / n) \sum_{i=1}^{n} a_{i}^{r}\right)^{1 / r}$. Then $P_{n}(r) / P_{n+m}(r) \geq a_{n} / a_{n+m}$. The lower bound is the best possible.

2000 Mathematics Subject Classification: 26D15.

1. Introduction. It is well known that the inequality

$$
\frac{n}{n+1}<\left(\frac{(1 / n) \sum_{i=1}^{n} i^{r}}{(1 /(n+1)) \sum_{i=1}^{n+1} i^{r}}\right)^{1 / r}<\frac{\sqrt[n]{n !}}{\sqrt[n+1]{(n+1) !}}
$$

holds for $r>0$ and $n \in \mathbb{N}$. We call the left-hand side of this inequality Alzer's inequality [1] and the right-hand side Martins' inequality [8].

Let $\left\{a_{i}\right\}_{i \in \mathbb{N}}$ be a positive sequence. If $a_{i+1} a_{i-1} \geq a_{i}^{2}$ for $i \geq 2$, we call $\left\{a_{i}\right\}_{i \in \mathbb{N}}$ a logarithmically convex sequence; if $a_{i+1} a_{i-1} \leq a_{i}^{2}$ for $i \geq 2$, we call $\left\{a_{i}\right\}_{i \in \mathbb{N}}$ a logarithmically concave sequence.

In [2], Martins' inequality was generalized as follows: let $\left\{a_{i}\right\}_{i \in \mathbb{N}}$ be an increasing, logarithmically concave, positive, and nonconstant sequence satisfying $\left(a_{\ell+1} / a_{\ell}\right)^{\ell} \geq$ $\left(a_{\ell} / a_{\ell-1}\right)^{\ell-1}$ for any positive integer $\ell>1$, then

$$
\left(\frac{(1 / n) \sum_{i=1}^{n} a_{i}^{r}}{(1 /(n+m)) \sum_{i=1}^{n+m} a_{i}^{r}}\right)^{1 / r}<\frac{\sqrt[n]{a_{n} !}}{\sqrt[n+m]{a_{n+m} !}}
$$

where $r$ is a positive number, $n, m \in \mathbb{N}$, and $a_{i}$ ! denotes the sequence factorial $\prod_{i=1}^{n} a_{i}$. The upper bound is the best possible.

Recently, in [13], another generalization of Martins' inequality was obtained: let $n, m \in \mathbb{N}$ and let $\left\{a_{i}\right\}_{i=1}^{n+m}$ be an increasing, logarithmically concave, positive, and nonconstant sequence such that the sequence $\left\{i\left[a_{i+1} / a_{i}-1\right]\right\}_{i=1}^{n+m-1}$ is increasing. Then inequality (1.2) between ratios of the power means and of the geometric means holds. The upper bound is the best possible.

Alzer's inequality has invoked the interest of several mathematicians including, for example, Cerone et al. [3], Elezović and Pečarić [4], Guo [5, 16, 17], Kuang [6], Debnath [15], Liu [7], Luo [18], Ozeki [9], Sándor [19, 20], Ume [21], the first author [10, 11, 12, 14] of this note, and so on. 
In [22], a general form of Alzer's inequality was obtained: let $\left\{a_{i}\right\}_{i=1}^{\infty}$ be a strictly increasing positive sequence and let $m$ be a natural number. If $\left\{a_{i}\right\}_{i=1}^{\infty}$ is logarithmically concave and the sequence $\left\{\left(a_{i+1} / a_{i}\right)^{i}\right\}_{i=1}^{\infty}$ is increasing, then

$$
\frac{a_{n}}{a_{n+m}}<\left(\frac{(1 / n) \sum_{i=1}^{n} a_{i}^{r}}{(1 /(n+m)) \sum_{i=1}^{n+m} a_{i}^{r}}\right)^{1 / r}
$$

In this short note, utilizing the mathematical induction, we obtain the following theorem.

THEOREM 1.1. Let $n$ and $m$ be natural numbers. Suppose that $\left\{a_{i}\right\}_{i=1}^{n+m}$ is an increasing, logarithmically convex, and positive sequence. Denote the power mean $P_{n}(r)$ for any given positive real number $r$ by

$$
P_{n}(r)=\left(\frac{1}{n} \sum_{i=1}^{n} a_{i}^{r}\right)^{1 / r}
$$

Then the sequence $\left\{P_{i}(r) / a_{i}\right\}_{i=1}^{n+m}$ is decreasing for any given positive real number $r$, that is,

$$
\frac{P_{n}(r)}{P_{n+m}(r)} \geq \frac{a_{n}}{a_{n+m}}
$$

The lower bound in (1.5) is the best possible.

Considering that the exponential functions $a^{x^{\alpha}}$ and $a^{\alpha^{x}}$ for given constants $\alpha \geq 1$ and $a>1$ are logarithmically convex on [0, ), as a corollary of Theorem 1.1 , we have the following corollary.

COROLLARY 1.2. Let $\alpha \geq 1$ and $a>1$ be two constants. For any given real number $r$, the following inequalities hold:

$$
\begin{aligned}
\frac{a^{(n+k)^{\alpha}}}{a^{(n+m+k)^{\alpha}}} & \leq\left(\frac{(1 / n) \sum_{i=k+1}^{n+k} a^{i^{\alpha} r}}{(1 /(n+m)) \sum_{i=k+1}^{n+m+k} a^{i^{\alpha^{\alpha}}}}\right)^{1 / r}, \\
\frac{a^{\alpha^{n+k}}}{a^{\alpha^{n+m+k}}} & \leq\left(\frac{(1 / n) \sum_{i=k+1}^{n+k} a^{\alpha^{i} r}}{(1 /(n+m)) \sum_{i=k+1}^{n+m+k} a^{\alpha^{i} r}}\right)^{1 / r},
\end{aligned}
$$

where $n$ and $m$ are natural numbers and $k$ is a nonnegative integer. The lower bounds above are the best possible.

2. Proof of Theorem 1.1. Inequality (1.5) is equivalent to

$$
\frac{(1 / n) \sum_{i=1}^{n} a_{i}^{r}}{(1 /(n+m)) \sum_{i=1}^{n+m} a_{i}^{r}} \geq \frac{a_{n}^{r}}{a_{n+m}^{r}},
$$


that is,

$$
\frac{1}{(n+m) a_{n+m}^{r}} \sum_{i=1}^{n+m} a_{i}^{r} \leq \frac{1}{n a_{n}^{r}} \sum_{i=1}^{n} a_{i}^{r} .
$$

This is also equivalent to

$$
\frac{1}{(n+1) a_{n+1}^{r}} \sum_{i=1}^{n+1} a_{i}^{r} \leq \frac{1}{n a_{n}^{r}} \sum_{i=1}^{n} a_{i}^{r} .
$$

Since

$$
\sum_{i=1}^{n+1} a_{i}^{r}=\sum_{i=1}^{n} a_{i}^{r}+a_{n+1}^{r}
$$

inequality (2.3) reduces to

$$
\sum_{i=1}^{n} a_{i}^{r} \geq \frac{n a_{n}^{r} a_{n+1}^{r}}{(n+1) a_{n+1}^{r}-n a_{n}^{r}}
$$

It is easy to see that inequality (2.5) holds for $n=1$.

Assume that inequality (2.5) holds for some $n>1$. Using the principle of mathematical induction and considering equality (2.4) and the inductive hypothesis, it is easy to show that the induction for inequality (2.5) on $n+1$ can be written as

$$
\frac{(n+2) a_{n+2}^{r}-(n+1) a_{n+1}^{r}}{(n+1) a_{n+1}^{r}-n a_{n}^{r}} \geq\left(\frac{a_{n+2}}{a_{n+1}}\right)^{r},
$$

which can be rearranged as

$$
n\left[\left(\frac{a_{n+1}}{a_{n+2}}\right)^{r}-\left(\frac{a_{n}}{a_{n+1}}\right)^{r}\right]+\left(\frac{a_{n+1}}{a_{n+2}}\right)^{r} \leq 1 .
$$

Since the sequence $\left\{a_{i}\right\}_{i=1}^{n+m}$ is increasing, we have $a_{n+1} / a_{n+2} \leq 1$ and $\left(a_{n+1} / a_{n+2}\right)^{r} \leq$ 1. From the logarithmical convexity of the sequence $\left\{a_{i}\right\}_{i=1}^{n+m}$, it follows that $a_{n+1} / a_{n+2}$ $\leq a_{n} / a_{n+1}$ and $\left(a_{n+1} / a_{n+2}\right)^{r}-\left(a_{n} / a_{n+1}\right)^{r} \leq 0$. Therefore, inequality (2.7) is valid. Thus, inequality (1.5) holds.

It can be easily shown by L'Hospital rule that

$$
\lim _{r \rightarrow \infty} \frac{P_{n}(r)}{P_{n+m}(r)}=\frac{a_{n}}{a_{n+m}}
$$

Hence, the lower bound in (1.5) is the best possible. The proof is complete. 
ACKNOWLEDGMENTS. The first two authors were supported in part by the National Natural Science Foundation of China (NNSF) Grant 10001016, SF for the Prominent Youth of Henan Province Grant 0112000200, SF of Henan Innovation Talents at Universities, Natural Science Foundation (NSF) of Henan Province Grant 004051800, and Doctor Fund of Jiaozuo Institute of Technology, China. The third author was partially supported by a grant from the Faculty Research Council of the University of Texas-Pan American.

\section{REFERENCES}

[1] H. Alzer, On an inequality of H. Minc and L. Sathre, J. Math. Anal. Appl. 179 (1993), no. 2, 396-402.

[2] T. H. Chan, P. Gao, and F. Qi, On a generalization of Martins' inequality, Monatsh. Math. 138 (2003), no. 3, 179-187, RGMIA Res. Rep. Coll. 4 (2001), no. 1, Article 12, 93-101.

[3] Ch.-P. Chen, F. Qi, P. Cerone, and S. S. Dragomir, Monotonicity of sequences involving convex and concave functions, Math. Inequal. Appl. 6 (2003), no. 2, 229-239, RGMIA Res. Rep. Coll. 5 (2002), no. 1, Article 1, 3-13.

[4] N. Elezović and J. Pečarić, On Alzer's inequality, J. Math. Anal. Appl. 223 (1998), no. 1, 366-369.

[5] B.-N. Guo and F. Qi, An algebraic inequality, II, RGMIA Res. Rep. Coll. 4 (2001), no. 1, Article 8, 55-61.

[6] J.-Ch. Kuang, Some extensions and refinements of Minc-Sathre inequality, Math. Gaz. 83 (1999), 123-127.

[7] Zh. Liu, New generalization of H. Alzer's inequality, Tamkang J. Math. 34 (2003), no. 3, 255-260.

[8] J. S. Martins, Arithmetic and geometric means, an application to Lorentz sequence spaces, Math. Nachr. 139 (1988), 281-288.

[9] N. Ozeki, On some inequalities, J. College Arts Sci. Chiba Univ. 4 (1965), no. 3, 211-214.

[10] F. Qi, Generalization of H. Alzer's inequality, J. Math. Anal. Appl. 240 (1999), no. 1, 294297.

[11] _ Generalizations of Alzer's and Kuang's inequality, Tamkang J. Math. 31 (2000), no. 3, 223-227, RGMIA Res. Rep. Coll. 2 (1999), no. 6, Article 12, 891-895.

[12] _ An algebraic inequality, J. Inequal. Pure Appl. Math. 2 (2001), no. 1, Article 13, 1-3, RGMIA Res. Rep. Coll. 2 (1999), no. 1, Article 8, 81-83.

[13] _ On a new generalization of Martins' inequality, RGMIA Res. Rep. Coll. 5 (2002), no. 3, Article 13, 527-538.

[14] _ Inequalities and monotonicity of sequences involving $\sqrt[n]{(n+k) ! / k !}$, Soochow J. Math. 29 (2004), no. 4, 353-361, RGMIA Res. Rep. Coll. 2 (1999), no. 5, Article 8, 685-692.

[15] F. Qi and L. Debnath, On a new generalization of Alzer's inequality, Int. J. Math. Math. Sci. 23 (2000), no. 12, 815-818.

[16] F. Qi and B.-N. Guo, Monotonicity of sequences involving convex function and sequence, RGMIA Res. Rep. Coll. 3 (2002), no. 2, Article 14, 321-329.

[17] _ An inequality between ratio of the extended logarithmic means and ratio of the exponential means, Taiwanese J. Math. 7 (2003), no. 2, 229-237.

[18] F. Qi and Q.-M. Luo, Generalization of H. Minc and J. Sathre's inequality, Tamkang J. Math. 31 (2000), no. 2, 145-148, RGMIA Res. Rep. Coll. 2 (1999), no. 6, Article 14, 909-912.

[19] J. Sándor, On an inequality of Alzer, J. Math. Anal. Appl. 192 (1995), no. 3, 1034-1035.

[20] Comments an inequality for the sum of powers of positive numbers, RGMIA Res. Rep. Coll. 2 (1999), no. 2, 259-261.

[21] J. S. Ume, An elementary proof of H. Alzer's inequality, Math. Japon. 44 (1996), no. 3, 521522. 
[22] Z. Xu and D. Xu, A general form of Alzer's inequality, Comput. Math. Appl. 44 (2002), no. 3-4, 365-373.

Feng Qi: Department of Applied Mathematics and Informatics, Jiaozuo Institute of Technology, Jiaozuo City, Henan 454000, China

E-mail address: qifeng@jzit.edu.cn; fengqi618@member.ams.org

URL: http://rgmia.vu.edu.au/qi .htm1

Bai-Ni Guo: Department of Applied Mathematics and Informatics, Jiaozuo Institute of Technology, Jiaozuo City, Henan 454000, China

E-mail address: guobaini@jzit.edu.cn

Lokenath Debnath: Department of Mathematics, University of Texas-Pan American, Edinburg, TX 78539, USA

E-mail address: debnath1@panam.edu 


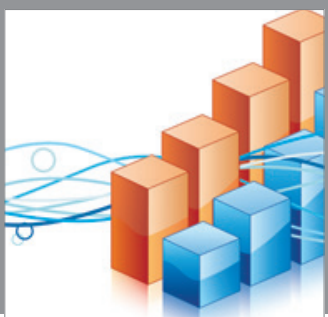

Advances in

Operations Research

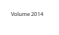

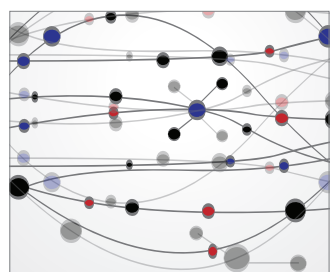

\section{The Scientific} World Journal
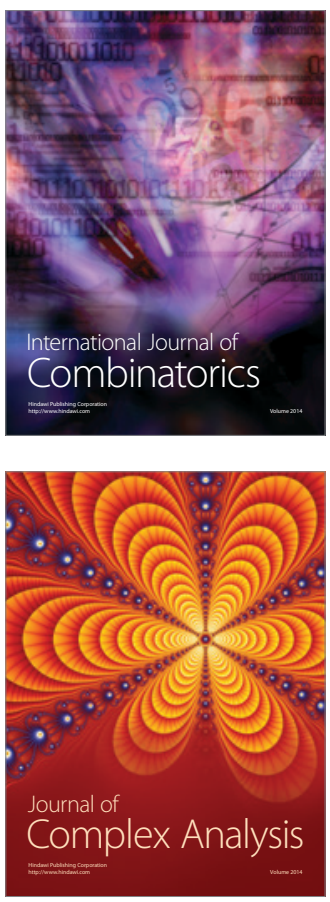

International Journal of

Mathematics and

Mathematical

Sciences
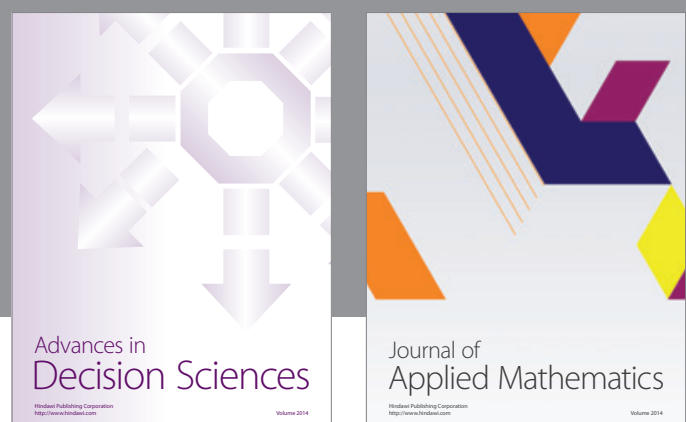

Journal of

Applied Mathematics
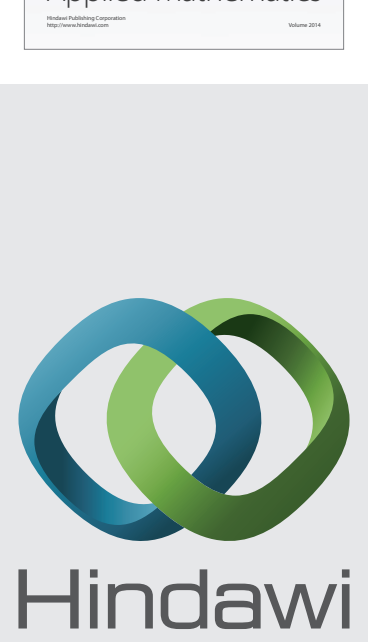

Submit your manuscripts at http://www.hindawi.com
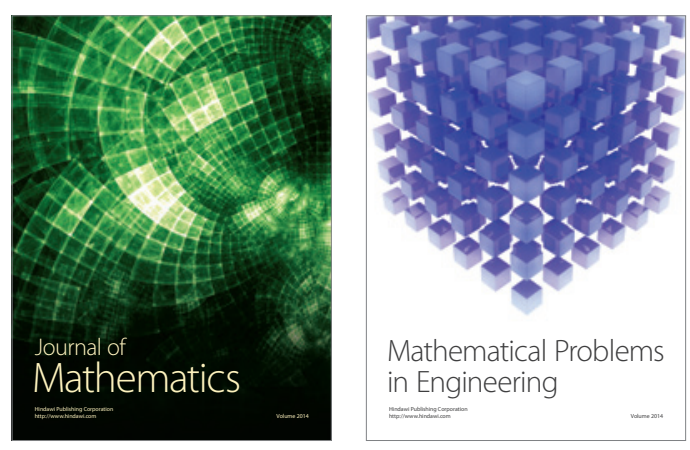

Mathematical Problems in Engineering
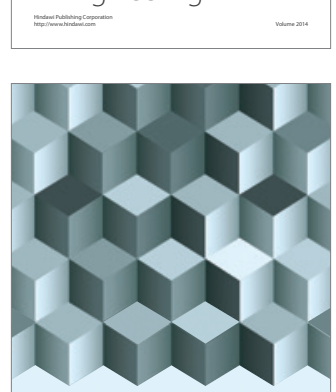

Journal of

Function Spaces
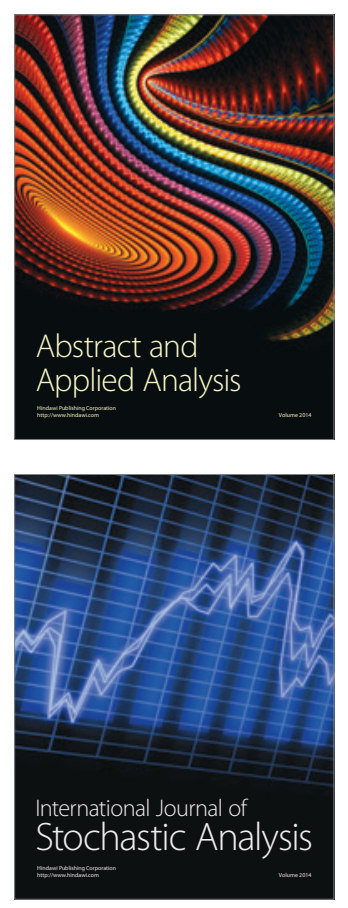

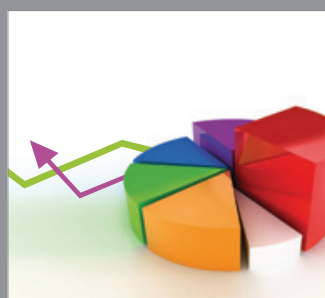

ournal of

Probability and Statistics

Promensencen
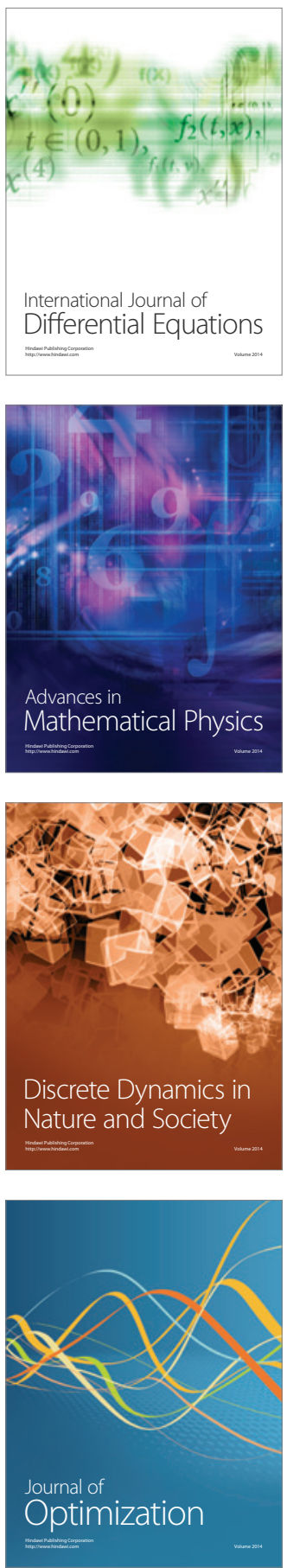